\title{
Relationship of immunodiagnostic assays for tuberculosis and numbers of circulating CD4+ T-cells in HIV infection
}

\author{
L. Leidl*,**, H. Mayanja-Kizza",**, G. Sotgiu๗, J. Baseke ${ }^{\#}$, M. Ernst $^{+}$, C. Hirsch $^{\S}$, \\ D. Goletti ${ }^{\dagger}$, Z. Toossi $i^{\S}$ and C. Lange*
}

ABSTRACT: Infection with HIV is the greatest risk factor for tuberculosis (TB) in Africa. Tuberculin skin test (TST), QuantiFERON-TB Gold In-Tube (QFT-G-IT) and T-Spot.TB assays were performed in newly diagnosed HIV-infected individuals with and without active TB and in HIV-uninfected subjects at a university outpatient clinic in Kampala, Uganda.

A total of 135 individuals were enrolled: 109 with a new diagnosis of HIV-1 infection but no active TB, 19 with HIV-1 infection and active TB, and seven HIV-uninfected healthy subjects. In control subjects immune responses were positive in $57.2 \%$ by TST and in $100 \%$ by at least one interferon- $\gamma$ release assay.

In HIV-1 infected patients without active TB, induration in the TST $(\mathrm{mm})(\mathrm{rho}=0.41$, $\mathrm{p}$-value $<0.0001$ ) and concentration of interferon (IFN)- $\gamma$ in the QFT-G-IT tube with Mycobacterium tuberculosis-specific antigens ( $r h o=0.38 ; p=0.0001)$ were negatively correlated to numbers of circulating CD4+ T-cells, while numbers of IFN- $\gamma$ producing cells (rho $=0.03-0.13$; $p$-value $=0.21-$ 0.77 ) and frequencies of positive test results for the T-Spot.TB test among groups of patients with different levels of immunodeficiency remained constant $(p$-value $\mathbf{0 . 4 6}$ ).

In HIV-1 infection, TST and QFT-G-IT immune responses are both strongly related to the degree of immunodeficiency, while results of the T-Spot.TB are independent of the level of CD4+ T-cell depletion.

KEYWORDS: Culture filtrate protein-10, early secretory antigenic target-6, HIV, interferon- $\gamma$ release assay, latent tuberculosis infection, tuberculosis

ccording to a previous estimate by the World Health Organization, approximately one third of the world population is infected with Mycobacterium tuberculosis [1]. Latent tuberculosis infection (LTBI) is usually defined as infection with $M$. tuberculosis complex, manifested by a pre-defined tuberculin skin test (TST) reaction and/or positive interferon- $\gamma$ release assay (IGRA) without any sign of active disease [2]. As a result of a successful immune response, only a minority of those individuals who are thought to have become latently infected with $M$. tuberculosis ever develop tuberculosis (TB) $[3,4]$. Nevertheless, approximately 9 million people are diagnosed with active TB and nearly 2 million die of the disease annually [5].

TB and infection with HIV are leading public health problems in Africa [6]. A dangerous interaction of these two diseases has been documented and includes findings of increased morbidity and mortality in dually infected subjects [7]. In fact, TB continues to be the leading cause of death in individuals with HIV-infection [8]. Recent data also indicate that infection with HIV doubles the risk of TB reactivation early after HIV seroconversion [9]. Furthermore, in HIVinfected individuals with LTBI the annual risk of developing active TB is $5-10 \%$ [10]. Thus, rapid identification and preventive treatment of HIVinfected individuals with LTBI, who are at risk of developing active $\mathrm{TB}$, is a priority in the fight against TB in Africa [11].

However, achieving this goal has been hampered to date by limitations in TST reactivity, particularly in the majority of individuals with advanced HIV-1 infection and presumptive LTBI [12-15] IGRAs may prove to be more reliable for the diagnosis of LTBI in individuals with HIV

For editorial comments see page 475 .

AFFILIATIONS

Divisons of ${ }^{*}$ Clinical Infectious Diseases, and

+Immune Cell-Analytics, Research Center Borstel, Borstel, Germany. \# Institute of Infectious Diseases, Makerere University, Kampala, Uganda.

"Hygiene and Preventive Medicine Institute, Sassari University, Sassari, ${ }^{f}$ Translational Research Unit, National Institute for Infectious Diseases L. Spallanzani, Rome, Italy ${ }^{\S}$ Tuberculosis Research Unit, Case Western Reserve University, Cleveland, $\mathrm{OH}$, USA.

**Both authors contributed equally to this study.

CORRESPONDENCE

C. Lange

Research Center Borstel

Parkallee 35

D-23845 Borstel

Germany

E-mail: clange@łz-borstel.de

Received:

March 202009

Accepted after revision:

July 072009

First published online:

July 162009 
infection [16-21]. Nevertheless, studies comparing TST with the two commercially available IGRAs, the QuantiFERON-TB Gold In-Tube (Cellestis, Melbourne, Australia) and the TSpot.TB (Oxford Immunotec, Abingdon, UK) in a country of high HIV prevalence and endemic for TB are limited [21].

To evaluate current methods for the immunodiagnosis of LTBI and understand their relationship with the level of immunodeficiency in patients with HIV infection, we compared the performance of different IGRAs and the TST in an African country with high TB-incidence.

\section{MATERIAL AND METHODS}

\section{Study subjects and design}

The study was conducted in Kampala, Uganda, at the HIV outpatient clinic of the Infectious Diseases Institute of Makerere University. In 2006, the incidence of TB in Uganda was 355 per 100,000 . In recent years, $\sim 60 \%$ of TB patients tested for HIV in Uganda were HIV positive [22]; however, current HIVprevalence rates in patients with TB are estimated to be lower at 45-50\% (H. Mayanja-Kizza; personal communication).

The study was approved by the ethical committees of Makerere University (Uganda), Case Western Reserve University (Cleveland, OH, USA) and Lübeck University (Lübeck, Germany). All subjects provided written informed consent, consistent with good clinical practice and ethical guidelines of the US Department of Health and Human Services.

Following voluntary counselling and testing for HIV-1 infection, adult residents of Kampala who were referred to the Infectious Diseases Institute at Makerere University were invited to participate. All study subjects were antiretroviral therapy naïve. Prior TB, isoniazid preventive therapy, steroid therapy, pregnancy and, in HIV-infected people, a Karnofsky score $\leqslant 60$ or current opportunistic infection were exclusion criteria. The presence of any one of the symptoms cough, chest pain, recent weight loss, night sweats, fever, loss of appetite, swelling of lymph nodes or generalised tiredness triggered referral to the Tuberculosis Research Unit clinic where sputum examinations where performed to rule out active TB. All individuals were clinically examined and received chest radiography to rule out active pulmonary TB. Healthy HIVseronegative controls (staff of the Infectious Diseases Institute at Makerere University) where also enrolled.

HIV-seropositive individuals without risk factors for active TB were recruited at the National Institute for Infectious Diseases L. Spallanzani (Rome, Italy) as additional controls.

\section{TST}

At the first study visit, blood was taken and a $2 \mathrm{TU} / 0.1 \mathrm{~mL}$ TST (RT23; Statens Serum Institute, Copenhagen, Denmark) was placed on the volar aspect of the forearm, and the site was marked with a circle by felt-tip pen. At $48 \mathrm{~h}$, the transverse diameter of TST induration was determined by the ball-point pen and ruler method [23].

\section{IGRAs}

T-Spot.TB (Oxford Immunotec) and QuantiFERON-TB Gold in tube (Cellestis) were performed according to the manufacturer's guidelines.

\section{T-Spot.TB assay}

For the T-Spot.TB assays, peripheral blood mononuclear cells (PBMCs) were separated from heparinised venous blood by centrifugation and the interferon (IFN)- $\gamma$ ELISpot kit with a 96well plate was used. 250,000 cells per well were either stimulated with phytohaemagglutinin (positive control), left unstimulated (negative control) or contained peptides of early secretory antigenic target (ESAT)- 6 and culture filtrate protein (CFP)-10. After incubation for $18 \mathrm{~h}$, plates were developed according to the manufacturer's protocol and were read on an Immunospot Analyzer (AID, Strassberg, Germany). A positive result was defined as spot count in the ESAT-6 and/or CFP-10 panel minus spot count in the nil-control panel of at least five spots; a negative result was defined as less than five spots. Results were defined as indeterminate, if $>10$ spot-forming cells (sfc) were present in the nil-control wells (high background) and/or $<20 \mathrm{sfc}$ in the mitogen-positive control wells.

\section{QFT-G-IT assay}

For the QFT-G-IT test three 1-mL blood venous blood samples were collected in three evacuated tubes that were pre-coated with M. tuberculosis specific antigens (ESAT-6, CFP-10 and TB7.7) for the test, phytohaemagglutinin for the positive control, or no antigen for the negative control. Specimens were processed according to the manufacturer's instructions. Results were considered to be positive if the level of IFN- $\gamma$ in the ESAT-6, CFP-10 and TB7.7 antigen-exposed sample minus the level in the negative control was $\geqslant 0.35 \mathrm{IU} \cdot \mathrm{mL}^{-1}$ and $\geqslant 25 \%$ of the IFN- $\gamma$ concentration in the negative-control plasma. Indeterminate results were defined as either an unprovoked IFN- $\gamma$ level of $\geqslant 8.0 \mathrm{IU} \cdot \mathrm{mL}^{-1}$ in the negative-control plasma or an IFN- $\gamma$ response of $\leqslant 0.5 \mathrm{IU} \cdot \mathrm{mL}^{-1}$ on phytohaemagglutinin stimulation with a level of IFN- $\gamma$ in the TB antigen-exposed sample minus the level in the negative control of either $<0.35 \mathrm{IU} \cdot \mathrm{mL}^{1}$ or $<25 \%$ of the IFN $-\gamma$ concentration in the negative-control plasma.

\section{Laboratory evaluation}

Plasma HIV viral load was examined by Amplicor (Roche Diagnostics, Basel, Switzerland) and blood CD4+ cell counts were detected by flow cytometry (Becton Dickinson Inc., San Jose, CA, USA).

\section{Statistical analysis}

Data were analysed using Stata 9.0 (StataCorp; Stata Statistical Software Release 9, College Station, TX, USA). Assay responses were evaluated as continuous variables and as categorical variables (positive or negative responses). Comparisons between proportions were performed using Fisher's exact test; an unpaired t-test was used for continuous measurements to test relationships in unpaired analysis, while the Wilcoxon Mann-Whitney test was used when assumed that the dependent variable is a normally distributed interval variable. Group means $(>2)$ were compared using ANOVA; its nonparametric version (Kruskal-Wallis test) was used when appropriate. Test concordance was assessed by $\kappa$-statistics with agreement considered "slight" for $k \leqslant 0.20$, "fair" for $0.20<\mathrm{k} \leqslant 0.40$, "moderate" for $0.40<\mathrm{k} \leqslant 0.60$, "substantial" for $0.60<\mathrm{k} \leqslant 0.80$ and "optimal" for $0.80<\mathrm{k} \leqslant 1.00$ All analyses were two-sided. A p-value of 0.05 was considered significant $[24,25]$. 


\section{RESULTS}

Of the 190 individuals initially evaluated, 135 individuals met the inclusion/exclusion criteria and were included in the analysis. Of these, 109 were newly diagnosed HIV-1 positive patients with no signs or symptoms of active TB, 19 were newly diagnosed HIV-1 positive patients with active TB and seven were HIV-uninfected healthy controls. The study design, the demographic and clinical characteristics of the study participants and results of the immunodiagnostic assays are shown in figure 1 and table 1, respectively.

The median (interquartile range; IQR) level of circulating CD4 +T-cells was 182 (118) cells $\mu \mathrm{L}^{-}{ }^{1}$ and 283 (226)) cells $\cdot \mu \mathrm{L}^{-}{ }^{1}$ in HIV-infected patients with and without active TB, respectively. In $109 \mathrm{HIV}$-infected patients without active TB, median (IQR) levels of circulating CD4+ T-cells were 64 (43) cells $\mu \mathrm{LL}^{-}{ }^{1}, 179$ (68)) cells $\cdot \mu \mathrm{L}^{-}{ }^{1}$ and $\left.368(206)\right)$ cells $\cdot \mu \mathrm{L}^{-}{ }^{1}$ in subjects with CD4+ T-cell counts of $<100(n=10), 100-250 \quad(n=33)$ and $>250$ $(\mathrm{n}=66)$ ) cells $\cdot \mu \mathrm{L}^{-}{ }^{1}$, respectively. HIV-viral load was inversely related to $\mathrm{CD} 4+\mathrm{T}$-cell counts as expected $(\mathrm{p}<0.01)$ (table 1$)$. Sex and age were equally distributed among patients from the different CD4+ T-cell count strata.

HIV-uninfected controls were younger (mean $25.7 \mathrm{yrs}$ ) than HIV-positive individuals (mean 33.4 and $34.1 \mathrm{yrs}$ in individuals with and without active $\mathrm{TB}$, respectively). TST results were not available for $20(18.3 \%)$ individuals from the group of HIV-infected persons without active TB, as individuals did not return for test reading. In patients with active TB, no TST was performed. All seven HIV-uninfected controls had a TST reaction of $\geqslant 5 \mathrm{~mm}$ induration and in six $(85.7 \%)$ and four (57.2\%) HIV-uninfected individuals the TST induration was $\geqslant 10 \mathrm{~mm}$ and $\geqslant 15 \mathrm{~mm}$, respectively. In the control group however, TST results corresponded to the Gaussian distribution, HIV-infected patients were mostly TST negative or only had small TST diameters (range 5-10 mm) (table 1). In HIV-infected patients, induration in the TST was directly correlated to numbers of circulating CD4+ T-cells (Spearmans rho $=0.41 ;$ p-value $<0.0001)$ (table 2$) .30$ (33.7\%) out of 89 HIV-infected patients without active TB had a TST induration of $0 \mathrm{~mm}$.

QFT-G-IT test and T-Spot.TB results were positive in all seven healthy controls and five (70\%) healthy controls, respectively. The HIV-infected individuals without TB were stratified according to CD4+ T-cells counts of $>250,100-250$ and $<100$ cells $\mu \mathrm{L}^{-1}$. Frequencies of positive results for QFT-G-IT test declined significantly from $77.3 \%$ to $66.7 \%$ and $10 \%$ $(\mathrm{p}<0.0001)$, respectively, while frequencies of positive test results for T-Spot.TB were $50 \%, 57.6 \%$ and $70 \%$, respectively, and were not significantly different from the frequency of positive T-Spot.TB results in the group of HIV-uninfected controls $(p=0.76)$ (table 1, fig. 2).

Indeterminate test results in QFT-G-IT and T-Spot.TB were observed in a total of three $(2.8 \%)$ and four $(3.7 \%)$ of the 109 HIV-infected patients without active TB, respectively. Indeterminate test results were not related to levels of

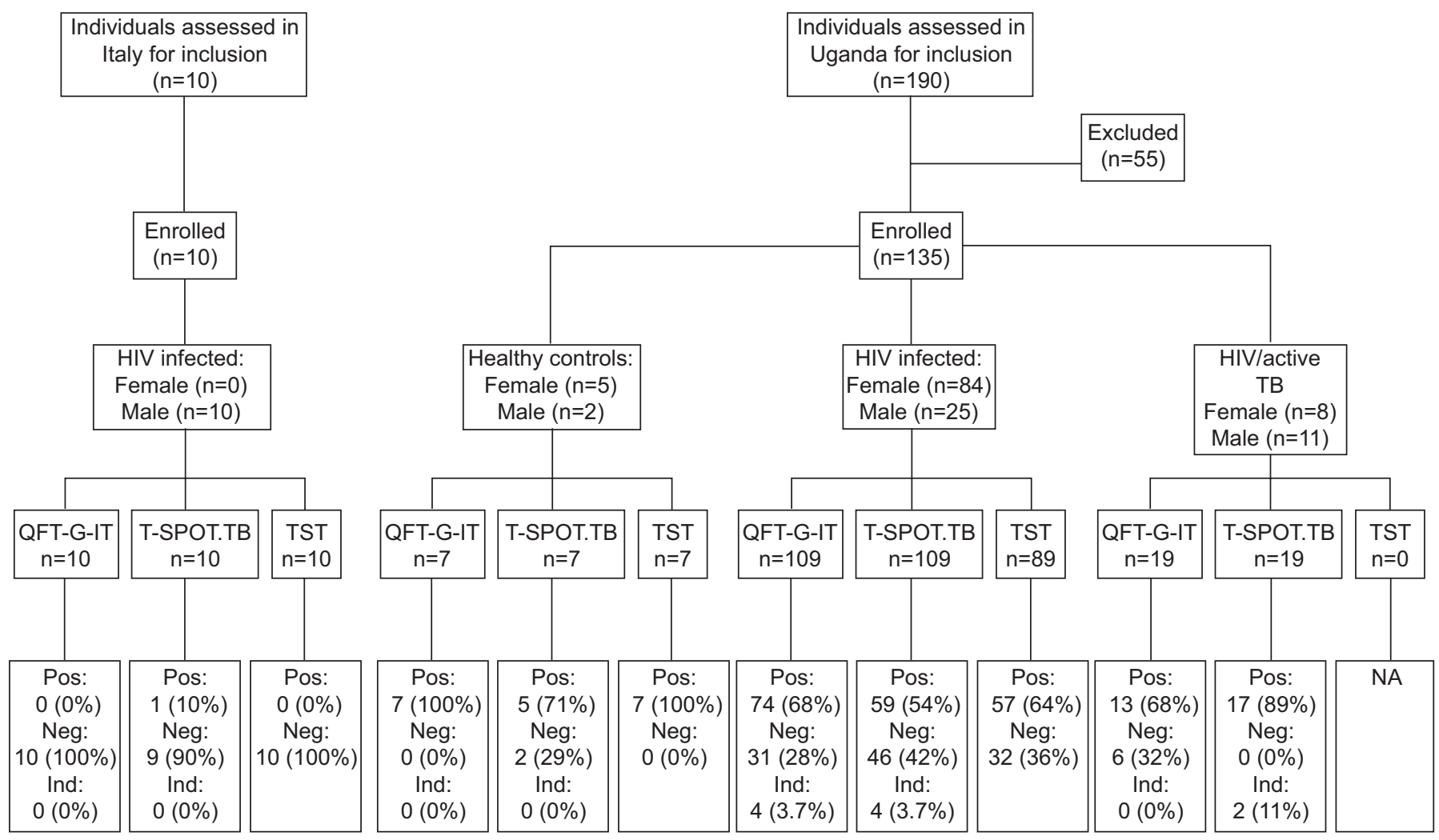

FIGURE 1. Flowchart of the study design and the main outcomes. TB: tuberculosis; QFT-G-IT: QuantiFERON-TB-Gold-In-Tube; TST: tuberculin skin test; Pos: positive; Neg: negative; Ind: indeterminate; NA: not applicable. 


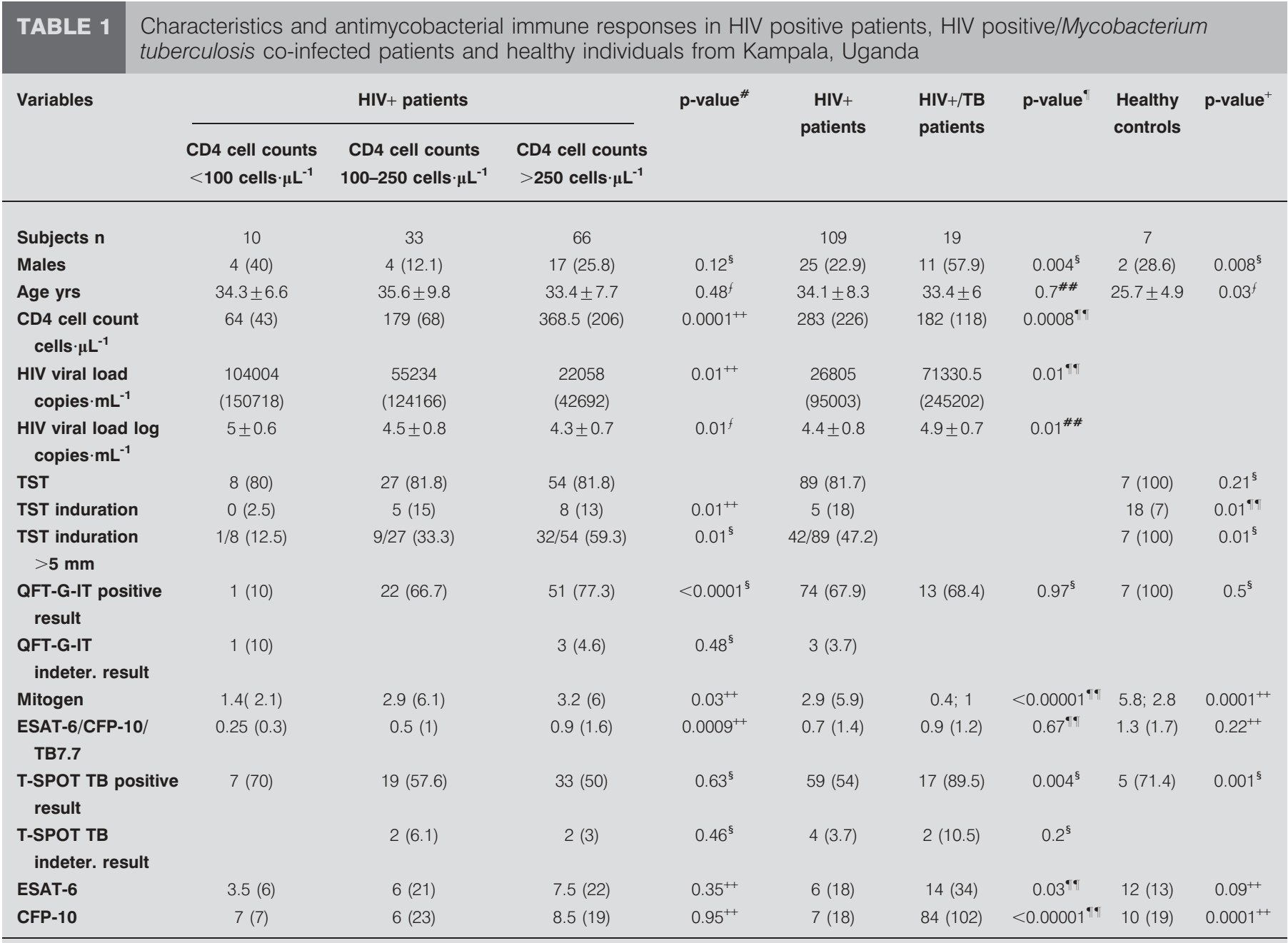

Data are presented as mean \pm SD, unless otherwise stated. Data for males, tuberculin skin test (TST) and all QuantiFERON-TB-Gold In-Tube (QFT-G-IT) and T-SPOT TB data are presented as n (\%). Data for CD4 cell count, HIV viral load copies, TST induration, mitogen, early secretaory antigenic target-6 (ESTAT-6) and culture filtrate protein-10 (CFP-10) are presented as median (interquartile range). TST induration $>5 \mathrm{~mm}$ is presented as $\mathrm{n} / \mathrm{N}(\%)$. indeter.: indeterminate. ${ }^{*}:$ comparison of the demographic and clinical characteristics among HIV+ patients with CD4 cell counts $<100$ cells $\cdot \mu \mathrm{L}^{-1}$, those with $100-250$ cells $\mu \mathrm{L}^{-1}$ and those with $>250 / \mu \mathrm{l}$; ${ }^{\circ}:$ comparison of the demographic and clinical characteristics among HIV+ patients and $\mathrm{HIV}+/ \mathrm{TB}$ co-infected patients; ${ }^{+}$: comparison of the demographic and clinical characteristics

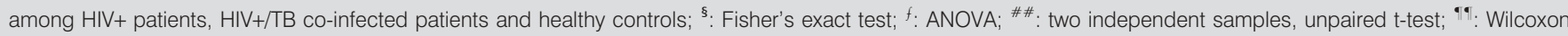
Mann-Whitney test; ${ }^{++}$: Kruskal-Wallis test.

circulating CD4+ T-cells ( $\mathrm{p}>0.45)$. In HIV-infected patients, the concentration of IFN- $\gamma$ in the tube with the specific antigens QFT-G-IT was directly correlated to numbers of circulating CD4+ T-cells (Spearman's rho $=0.38$; p-value $=0.0001$ ). In contrast, numbers of spots in the ESAT- 6 or CFP-10 antigen wells in the T-Spot.TB were not correlated to numbers of circulating CD4+ T-cells (Spearman's rho $=0.03$ and 0.13, respectively; $\mathrm{p}$-value $=0.77$ and 0.21 , respectively) (table 2).

Concordance between immunodiagnostic tests is shown in table 3. In the group of HIV-infected patients without active $\mathrm{TB}$, overall concordant test results between the T-Spot.TB and the QFT-G-IT, between the T-Spot.TB and the TST and between the QFT-G-IT and the TST were observed in $60.8 \% \quad(\kappa=0.17$; $\mathrm{SE}=0.09), 40.4 \%(\kappa=0.37 ; \mathrm{SE}=0.11)$ and $66.3 \%(\kappa=0.34 ; \mathrm{SE}=0.1)$, respectively. In HIV-infected individuals, overall test result concordance was best in the group of patients with $>250$ circulating CD4+ T-cells. In this group, highest concordance among test results was observed between the TST and the TSpot.TB $(73.1 \% ; \kappa=0.46 ; \mathrm{SE}=0.14)($ table 3$)$.

In patients with active TB, 17 (89\%) and two (11\%) out of 19 had a positive and indeterminate T-Spot.TB test result, respectively (fig. 1). Results of the QFT-G-IT were positive in $13(68 \%)$ and negative in six (32\%) of the 19 individuals with active $\mathrm{TB}$.

In 10 male HIV-seropositive individuals from Italy who were included as additional negative controls to document test specificity, the median (IQR) patient age was 41.4 (34-51) yrs, median (IQR) number of circulating CD4+ T-cell counts was 480 (303.5-778) cells $\mu \mathrm{L}^{-1}$. Results of the TST $(>5 \mathrm{~mm})$, the QTF-G-IT and the T-Spot.TB were positive in zero, zero and one patient, respectively. Indeterminate results in the 
TABLE 2 Correlation of numbers of circulating CD4+ T-cells with the skin induration in the TST, IFN-concentration in the ESAT-6, CFP-10 and TB 7.7 containing tube in the QuantiFERON-TB Gold In-Tube test and with numbers of sfc in response to incubation with ESAT-6 and CFP-10 in the T-Spot.TB test

Correlation of CD4+ T-cells per $\mu \mathrm{L}$

TST $\mathrm{mm}$

QuantiFERON-Gold-In Tube IFN- $\gamma$ concentration

T-SPOT.TB ESAT-6 sfC

T-SPOT.TB CFP-10 sfc

T-SPOT.TB highest value among ESAT-6/CFP-10 sfc

\section{Spearman's rho}

\subsection{1}

0.38

0.03

0.13

0.01 p-value

$<0.0001$

0.0001

0.77

0.21

0.31

Data in bold indicates statistically significant. TST: tuberculin skin test; IFN: interferon; ESAT-6: early secretory antigenic target-6; CFP-10: culture filtrate protein-10; sfc: spot-forming cells.

QTF-G-IT and T-Spot.TB assays were observed in no patients in both tests (fig. 1).

\section{DISCUSSION}

Combating co-infection of HIV with $M$. tuberculosis is a major goal of health care interventions in Africa [26]. TB is still the leading cause of HIV-related deaths in the developing world. Early identification and treatment of LTBI in individuals with HIV-infection is efficient [27] and important in order to reduce the spread of TB in Africa [28]. Detection of LTBI relies on immunodiagnostic tests, the TST and, more recently, IGRAs, but comparative data on their performance in individuals from high incidence countries of TB are still limited [20, 21].

In this study, HIV-infected individuals from a country of high incidence of TB were enrolled to directly compare the diagnostic accuracy of the TST, the T-Spot.TB test and the QFT-G-IT test for the diagnosis of LTBI. Results from this comparison add substantially to the current knowledge on the performance of these tests in individuals with different levels of immunodeficiency in HIV-infection. In a cohort of African

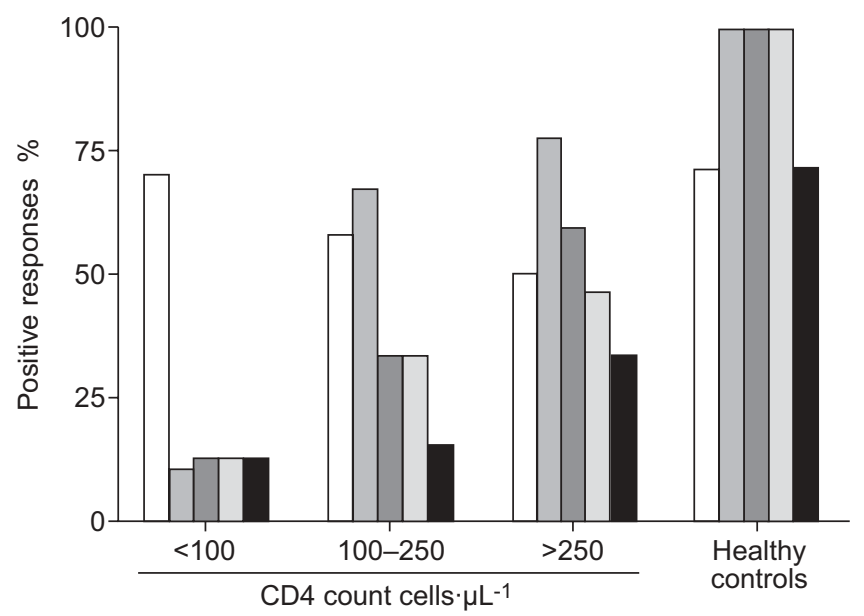

FIGURE 2. Frequencies of positive immune responses in HIV-infected persons from Kampala, Uganda, with $<100,100-250$ and $>250$ CD4+ T-cells and HIVuninfected controls in the T-Spot.TB test $(\square)$, QuantiFERON-TB Gold In-Tube test $(\square)$, and the tuberculin skin test induration cut-offs, $\geqslant 5 \mathrm{~mm}(\square), \geqslant 10 \mathrm{~mm}(\square)$ and $\geqslant 15 \mathrm{~mm}$ individuals from a community in Uganda, where TB is highly endemic, we found in patients without active TB that $57.2 \%$ of the HIV-uninfected individuals had a positive TST reaction of an induration of $>15 \mathrm{~mm}$, the recommended cut-off according to the American Thoracic Society criteria [29]. The TST induration in all HIV-uninfected controls was $>5 \mathrm{~mm}$, which is the recommended cut-off for HIV-infected individuals [29]. Only $12.5 \%$ of HIV-infected persons with $<100$ circulating CD4+ T-cells exhibited a positive TST reaction, and the diameter of TST induration was significantly correlated to numbers of circulating CD4+ T-cells in the HIV-infected individuals, as previously demonstrated [30].

All HIV-uninfected individuals without active TB had a positive reaction to one of the ex vivo IGRAs to test for memory against $M$. tuberculosis specific antigens, the QFT-G-IT or the T-Spot.TB test. Detection of high frequencies of LTBI in healthy individuals provided a basis to study $M$. tuberculosis specific immune responses in relation to the degree of immunosuppression in $\mathrm{HIV}$-infected individuals from the same community [31].

We found a striking difference in the frequencies of positive immune responses assayed by the different tests in HIVinfected individuals without active $\mathrm{TB}$ at different levels of circulating CD4+ T-cell counts. When we compared the size of TST induration and the concentration of IFN- $\gamma$ from the tubes containing the M. tuberculosis specific antigens ESAT-6, CFP-10 and TB7.7 in the QFT-G-IT, they correlated negatively to numbers of circulating CD4+ T-cells in individuals with HIVinfection. This correlation was not found for levels of antigen specific spots in the T-Spot.TB test. When we evaluated frequencies of positive immune responses in groups of patients with different levels of immunodeficiency, again the TST and QFT-G-IT were dependent upon the level of circulating CD4+ T-cell counts, while this relationship was not observed for the T-Spot.TB test. The highest frequency of positive TST and QFT-G-IT test results was observed in HIV-infected individuals with $>250 \mathrm{CD} 4+$ cells $\cdot \mu \mathrm{L}^{-1}$, indicating a dependency of these tests on the absolute number of circulating CD4+ T-cells. In contrast, frequencies of positive immune responses in the $\mathrm{T}$ Spot.TB test were independent of the level of immunodeficiency. The rate of positive T-Spot.TB results were comparable in HIV-infected patients with $<100,100-250$ and $>250$ CD4+ cells $\cdot \mu \mathrm{L}^{-1}$ and HIV-uninfected controls. 


\begin{tabular}{|c|c|c|c|c|c|}
\hline $\begin{array}{ll}\text { TABLE } 3 & \text { Concorda } \\
\text { healthy } \mathrm{cc}\end{array}$ & $\begin{array}{l}\text { en three tests fc } \\
\text { n Kampala, Ug }\end{array}$ & immunodiagnosi & tent tuberculosi & ection in HIV-in & ed patients and \\
\hline \multirow[t]{2}{*}{ Concordance } & \multicolumn{3}{|c|}{ HIV+ patients } & \multirow[t]{2}{*}{ HIV+ patients } & \multirow[t]{2}{*}{ Healthy controls } \\
\hline & $\begin{array}{l}\text { CD4 cell counts } \\
<100 \text { cells } \mu \mathrm{L}^{-1}\end{array}$ & $\begin{array}{l}\text { CD4 cell counts } \\
100-250 \text { cells } \cdot \mu L^{-1}\end{array}$ & $\begin{array}{l}\text { CD4 cell counts } \\
>250 \text { cells } \cdot \mu \mathrm{L}^{-1}\end{array}$ & & \\
\hline TST/T-SPOT.TB & $37.5(0.09 ; 0.15)$ & $68(0.39 ; 0.18)$ & $73.1(0.46 ; 0.14)$ & $40.4(0.37 ; 0.11)$ & $57.1(0.09 ; 0.36)$ \\
\hline TST/QuantiFERON & $71.4(-0.17 ; 0.38)$ & $55.6(0.22 ; 0.15)$ & $71.2(0.35 ; 0.12)$ & $66.3(0.34 ; 0.1)$ & $57.1(0 ; 0)^{\#}$ \\
\hline
\end{tabular}

Consequently, there is a high level of discordant results among immunodiagnostic tests for the detection of LTBI in HIVinfected individuals [21, 32-35]. In our study, overall concordance among the results of all three tests was not more than fair in HIV-infected individuals without active TB. Similar to the results of the previously mentioned study from Germany, the lowest agreement among test results was observed between the two IGRAs $(\kappa=0.17)$. Moderate test agreement was only observed among the TST and T-Spot.TB in patients with $>250$ circulating CD4+ T-cells $(\kappa=0.46)$.

Our results strongly support previous observations that show robustness of antigen-specific immune responses in the ELISpot assays, irrespective of T-cell stratification in patients with HIV-infection [20, 34, 36, 37]. In the largest comparable trial by RANGAKA et al. [21] who enrolled 74 HIV-infected and 86 HIV-uninfected individuals in a township in the Cape region of South Africa, frequencies of T-Spot.TB results in HIVinfected individuals with $>250 \mathrm{CD} 4+$ cells $\cdot \mu \mathrm{L}^{-1}$ were comparable to those of individuals with $<250 \mathrm{CD} 4+$ cells $\cdot \mu \mathrm{L}^{-1}$, while frequencies of QFT-G-IT test results were lower in patients with more advanced HIV-infection. However, results did not reach statistical significance in that study [21].

Recently, immunodiagnostic tests for LTBI were compared among HIV-infected individuals in Germany, a country of low TB incidence [34]. In agreement with the results obtained in this study, positive results of the T-Spot.TB were independent of the numbers of circulating CD4+ T-cells.

In contrast to these observations, the proportion of patients with a positive response to an in house $M$. tuberculosis specific ELISpot decreased with the numbers of circulating CD4+ cells in HIV-infected individuals from Dakar, Senegal [38]. This effect was particularly observed in individuals with circulating CD4+ T-cell counts of $<50$ cells $\mu \mathrm{L}^{-1}$, indicating that indeterminate or negative ELISpot test results for the immunodiagnosis of LTBI in severely immunosuppressed individuals need to be interpretated with caution.

The low frequency of positive results in the QFT-G-IT test in persons with advanced CD4+ T-cell depletion in our cohort was not explained by a high number of indeterminate results in this group. Frequencies of indeterminate results were only observed in $3.6 \%$ of HIV-infected individuals by either IGRA, independently of the circulating CD4+ T-cell count. These data differ from other studies where high numbers of indeterminate results were found by the QFT-G-IT assay [39, 40] or by "in house" tests of ELISpot assays [33,41]. This is probably due to the fact that in the present study, the group of patients with CD4+ T-cells $<100$ cells $\mu \mathrm{L}^{-1}$ was relatively small to have a profound effect on the number of indeterminate results, compared to other studies, and that the majority of the HIV+ individuals with circulating CD4+ T-cells $>100$ cells $\cdot \mu \mathrm{L}^{-1}$ did not have active disease. These individuals were, therefore, less prone to the immune deficiency induced by TB disease per se [42-44].

In the present study, we demonstrated that T-Spot.TB positive results were independent from CD4+ T-cell counts, differing from QFT-G-IT results. These data can be explained by the fact that the detrimental effects of lymphopaenia associated with the late stages of HIV disease in reducing the efficiency of IGRA tests can be overcome or reduced by the ELISpot procedure. In fact, ELISpot involves a normalisation of the input of PBMC used in the assays (250,000 PBMC are always plated in each well). Therefore, although QFT-G-IT produces easier logistic calculation in which the cell input is not normalised, it could suffer from a high rate of negative results among those HIV-infected patients with advanced immunodeficiency $[19,40,45,46]$ as also shown in this study.

The limitations of our study need to be addressed. While we performed the largest comparison of the three currently available immunodiagnostic tests for latent TB infection in HIV-infected individuals in a country of high TB incidence to date, numbers of HIV-infected individuals with $<100$ circulating CD4+ T-cells and numbers of HIV-uninfected controls were still relatively small in our study. However, the results are robust and are strengthened by different statistical methods, including a very high correlation between the magnitude of immune-responses in the TST and QFT-G-IT and the numbers of CD4+ T-cells in the blood. HIV-uninfected controls were younger than HIV-infected individuals, leading to a possible bias in frequencies of positive immune responses in this group. However, HIV-infected individuals were, on average, aged $<35 \mathrm{yrs}$ and it is not expected that age influenced the test results substantially.

There are now growing numbers of studies reporting cross sectional data on frequencies of immune responses by IGRA in immunocompromised individuals without active TB. While 
these data provide important information, longitudinal studies on the predictive values of IGRA for the development of active TB are now urgently needed [47].

In conclusion, in HIV-infection, immune responses in the TST and QFT-G-IT are both strongly related to the degree of immunodeficiency, while the T-Spot.TB seems to function independently of the level of CD4+ T-cell depletion.

\section{SUPPORT STATEMENT}

This study was supported by a personal grant to C. Lange from the HW. and J. Hector Foundation (Weinheim, Germany), and by National Institute of Health (Bethesda, MD, USA) grants HL 51636 to Z. Toossi and AI 70022 given to the Tuberculosis Research Unit, Case Western Reserve University (Cleveland, OH, USA). ELISA test kits (Quantiferon-TB Gold In-Tube; Cellestis Ltd., Carnegie, Australia) and ELISpot test kits (T-SPOT®.TB; Oxford Immunotec, Abingdon, UK) were kindly supplied by the manufacturers. The manufacturers of the ELISA test and the ELISpot test had no influence on the study design, participating clinicians and/or scientists, selection of the study centres, data collection, data analysis or the decision to publish the manuscript.

\section{STATEMENT OF INTEREST}

A statement of interest for C. Lange can be found at www.erj. ersjournals.com $/ \mathrm{misc} /$ statements.dtl

\section{REFERENCES}

1 World Health Organization. Global Tuberculosis Control 2008: Surveillance, Planning, Financing. World Health Organization, Geneva, 2008.

2 Mack U, Migliori GB, Sester M, et al. LTBI: latent tuberculosis infection or lasting immune response to Mycobacterium tuberculosis. A TBNET consensus statement. Eur Respir J 2009; 33: 956-973.

3 Corbett EL, Steketee RW, ter Kuile FO, et al. HIV-1/AIDS and the control of other infectious diseases in Africa. Lancet 2002; 359: 2177-2187.

4 Vynnycky E, Fine PE. The natural history of tuberculosis: the implications of age-dependent risks of disease and the role of reinfection. Epidemiol Infect 1997; 119: 183-201.

5 Dolin PJ, Raviglione MC, Kochi A. Global tuberculosis incidence and mortality during 1990-2000. Bull World Health Organ 1994; 72 213-220.

6 Maartens G, Wilkinson RJ. Seminar: tuberculosis. Lancet 2007; 370: 230-243.

7 Toossi Z. Virological and immunological impact of tuberculosis on human immunodeficiency virus type 1 disease. J Infect Dis 2003, 188: $1146-1155$

8 Bates I, Fenton C, Gruber J, et al. Vulnerability to malaria, tuberculosis, and HIV/AIDS infection and disease. Part 1: determinants operating at individual and household level. Lancet Infect Dis 2004; 4: 267-277.

9 Sonnenberg P, Glynn JR, Fielding K, et al. How soon after infection with HIV does the risk of tuberculosis start to increase? A retrospective cohort study in South African gold miners. J Infect Dis 2005; 191: 150-158.

10 Lawn SD, Badri M, Wood R. Tuberculosis among HIV-infected patients receiving HAART: long term incidence and risk factors in a South African cohort. Aids 2005; 19: 2109-2116.

11 Chaisson RE, Martinson NA. Tuberculosis in Africa: combating an HIV-driven crisis. N Engl J Med 2008; 358: 1089-1092.

12 Chin DP, Osmond D, Page-Shafer K. Reliability of anergy skin testing in persons with HIV infection. The pulmonary Complications of HIV Infection Study Group. Am J Respir Crit Care Med 1996; 153: 1982-1984.
13 Caiaffa WT, Graham NM, Galai N, et al. Instability of delayed-type hypersensitivity skin test anergy in human immunodeficiency virus infection. Arch Intern Med 1995; 155: 2111-2117.

14 Duncan LE, Elliott AM, Hayes RJ, et al. Tuberculin sensitivity and HIV-1 status of patients attending a sexually transmitted diseases clinic in Lusaka, Zambia: a cross-sectional study. Trans R Soc Trop Med Hyg 1995; 89: 37-40.

15 Markowitz N, Hansen NI, Wilcosky TC. Tuberculin and anergy testing in HIV-seropositive and HIV-seronegative persons. Pulmonary Complications of HIV Infection Study Group. Ann Intern Med 1993; 119: 185-193.

16 Chapman AL, Munkanta M, Wilkinson KA, et al. Rapid detection of active and latent tuberculosis infection in HIV-positive individuals by enumeration of Mycobacterium tuberculosis-specific T cells. Aids 2002; 16: 2285-2293.

17 Dheda K, Lalvani A, Miller RF, et al. Performance of a T-cell-based diagnostic test for tuberculosis infection in HIV-infected individuals is independent of CD4 cell count. Aids 2005; 19: 2038-2041.

18 Lalvani A, Nagvenkar P, Udwadia Z, et al. Enumeration of T cells specific for RD1-encoded antigens suggests a high prevalence of latent Mycobacterium tuberculosis infection in healthy urban Indians. J Infect Dis 2001; 183: 469-477.

19 Luetkemeyer AF, Charlebois ED, Flores LL, et al. Comparison of an interferon- $\gamma$ release assay with tuberculin skin testing in HIVinfected individuals. Am J Respir Crit Care Med 2007; 175: 737-742.

20 Lawn SD, Bangani N, Vogt M, et al. Utility of interferon- $\gamma$ ELISPOT assay responses in highly tuberculosis-exposed patients with advanced HIV infection in South Africa. BMC Infect Dis 2007; 7: 99.

21 Rangaka MX, Wilkinson KA, Seldon R, et al. Effect of HIV-1 infection on T-Cell-based and skin test detection of tuberculosis infection. Am J Respir Crit Care Med 2007; 175: 514-520.

22 World Health Organization. Global Tuberculosis Control: Uganda 2007. World Health Organization Geneva.

23 Sokal JE. Measurement of delayed skin-test responses. N Engl J Med 1975; 293: 501-502.

24 Landis JR, Koch GG. The measurement of observer agreement for categorical data. Biometrics 1977; 33: 159-174.

25 Altman DG. Practical statistics for medical research. Chapman and Hall, London, 1991.

26 Corbett EL, Watt CJ, Walker N, et al. The growing burden of tuberculosis: global trends and interactions with the HIV epidemic. Arch Intern Med 2003; 163: 1009-1021.

27 Perkins MD, Cunningham J. Facing the crisis: improving the diagnosis of tuberculosis in the HIV era. J Infect Dis 2007; 196: $15-27$.

28 Nunn P, Reid A, De Cock KM. TB and HIV Infection: the global setting. J Infect Dis 2007; 196: 5-14.

29 Lee JY, Choi HJ, Park IN, et al. Comparison of two commercial interferon- $\gamma$ assays for diagnosing Mycobacterium tuberculosis infection. Eur Respir J 2006; 28: 24-30

30 Antonucci G, Girardi E, Raviglione MC, et al. Risk factors for tuberculosis in HIV-infected persons. A prospective cohort study. The Gruppo Italiano di Studio Tubercolosi e AIDS (GISTA). JAMA 1995; 274: 143-148.

31 Sarrazin H, Wilkinson KA, Andersson J, et al. Association between tuberculin skin test reactivity, the memory CD4 cell subset, and circulating FoxP3-expressing cells in HIV-infected persons. J Infect Dis 2009; 199: 702-710.

32 Richeldi L, Losi M, D'Amico R, et al. Performance of tests for latent tuberculosis in different groups of immunocompromised patients. Chest 2009; 136: 198-204.

33 Talati NJ, Seybold U, Humphrey B, et al. Poor concordance between interferon- $\gamma$ release assays and tuberculin skin tests in diagnosis of latent tuberculosis infection among HIV-infected individuals. BMC Infect Dis 2009; 9: 15.

34 Stephan C, Wolf T, Goetsch U, et al. Comparing QuantiFERONtuberculosis gold, T-SPOT tuberculosis and tuberculin skin test in 
HIV-infected individuals from a low prevalence tuberculosis country. Aids 2008; 22: 2471-2479.

35 Mandalakas AM, Hesseling AC, Chegou NN, et al. High level of discordant IGRA results in HIV-infected adults and children. Int $J$ Tuberc Lung Dis 2008; 12: 417-423.

36 Day CL, Mkhwanazi N, Reddy S, et al. Detection of polyfunctional Mycobacterium tuberculosis-specific T cells and association with viral load in HIV-1-infected persons. J Infect Dis 2008; 197: 990-999.

37 Hammond AS, McConkey SJ, Hill PC, et al. Mycobacterial T cell responses in HIV-infected patients with advanced immunosuppression. J Infect Dis 2008; 197: 295-299.

38 Karam F, Mbow F, Fletcher H, et al. Sensitivity of IFN- $\gamma$ release assay to detect latent tuberculosis infection is retained in HIVinfected patients but dependent on HIV/AIDS progression. PLoS ONE 2008; 3: e1441.

39 Aabye MG, Ravn P, PrayGod G, et al. The impact of HIV infection and CD4 cell count on the performance of an interferon $\gamma$ release assay in patients with pulmonary tuberculosis. PLoS ONE 2009; 4: e4220.

40 Raby E, Moyo M, Devendra A, et al. The effects of HIV on the sensitivity of a whole blood IFN- $\gamma$ release assay in Zambian adults with active tuberculosis. PLOS ONE 2008; 3: e2489.

41 Vincenti D, Carrara S, Butera O, et al. Response to region of difference 1 (RD1) epitopes in human immunodeficiency virus
(HIV)-infected individuals enrolled with suspected active tuberculosis: a pilot study. Clin Exp Immunol 2007; 150: 91-98.

42 Hirsch CS, Ellner JJ, Blinkhorn R, et al. In vitro restoration of T cell responses in tuberculosis and augmentation of monocyte effector function against Mycobacterium tuberculosis by natural inhibitors of transforming growth factor beta. Proc Natl Acad Sci USA 1997; 94: 3926-3931.

43 Hirsch CS, Hussain R, Toossi Z, et al. Cross-modulation by transforming growth factor beta in human tuberculosis: suppression of antigen-driven blastogenesis and interferon $\gamma$ production. Proc Natl Acad Sci USA 1996; 93: 3193-3198.

44 Vanham G, Toossi Z, Hirsch CS, et al. Examining a paradox in the pathogenesis of human pulmonary tuberculosis: immune activation and suppression/anergy. Tuber Lung Dis 1997; 78: 145-158.

45 Brock I, Ruhwald M, Lundgren B, et al. Latent tuberculosis in HIV positive, diagnosed by the $M$. tuberculosis specific interferon- $\gamma$ test. Respir Res 2006; 7: 56.

46 Jones S, de Gijsel D, Wallach FR, et al. Utility of QuantiFERON-TB Gold in-tube testing for latent TB infection in HIV-infected individuals. Int J Tuberc Lung Dis 2007; 11: 1190-1195.

47 Hill PC, Jackson-Sillah DJ, Fox A, et al. Incidence of tuberculosis and the predictive value of ELISPOT and Mantoux tests in Gambian case contacts. PLoS ONE 2008; 3: e1379. 